\title{
Ag Nanoparticles: Experimental Study of Sign Identification of Nonlinear Refractive Index by Moiré Deflectometry and Z-Scan Methods
}

\author{
Adeleh Granmayeh Rad, ${ }^{1}$ Khosro Madanipour, ${ }^{2}$ and Ata Koohian ${ }^{3}$ \\ ${ }^{1}$ Department of Physics, Faculty of Sciences, Roudehen Branch, Islamic Azad University, Roudehen 3973188981, Iran \\ ${ }^{2}$ Optical Measurement Central Lab, Amirkabir University of Technology, Tehran 4413-15875, Iran \\ ${ }^{3}$ Department of Physics, University of Tehran, Tehran 1439955961, Iran
}

Correspondence should be addressed to Adeleh Granmayeh Rad; granmayeh@riau.ac.ir

Received 5 July 2013; Accepted 30 July 2013

Academic Editors: R. Azimirad and J. Shen

Copyright ( 2013 Adeleh Granmayeh Rad et al. This is an open access article distributed under the Creative Commons Attribution License, which permits unrestricted use, distribution, and reproduction in any medium, provided the original work is properly cited.

\begin{abstract}
Two different methods are presented for the sign identification of nonlinear refractive index $\left(n_{2}\right)$ of Ag colloidal nanoparticles which are based on nonscanning Moiré deflectometry and Z-scan. In the Moiré deflectometry setup, two lasers are used, one is used as pump laser which causes thermal nonlinear effects in the sample, and the second one is used as the probe laser for monitoring these effects by Moiré deflectometry system. By observing the deflected Moiré fringes, we can determine the sign of nonlinear refractive index in real time, and there would be no need for calibration or complicated calculations. The second technique for sign identification is Z-scan. In this technique, a CW $532 \mathrm{~nm}$ second harmonic Nd:YAG laser with a beam power of $55 \mathrm{~mW}$ is used as the excitation source. Results show that the nonlinear refractive index is negative for Ag nanoparticles in pure water by both methods.
\end{abstract}

\section{Introduction}

Measurements of nonlinear optical parameters of colloidal metallic nano-particles have drawn a lot of attentions because of their fast nonlinear optical response and high nonlinearity ability [1]. Colloidal metallic solutions are frequently used in the design of optical instruments and photonic limiters because of their photoinduced nonlinear properties [2, 3]. There are two standard and usual methods for sign identification of nonlinear refractive index of nanoparticles, Z-scan [4-7] and Moiré deflectometry techniques [8-13]. In Z-scan method, the refractive index and the sign are found by drawing the diagram of the $z$ dependence of the transmitted beam intensity but in Moiré deflectometry technique, these are found by observing the Moiré fringes patterns [14]. It has also been shown that Moiré method is simpler and more robust than other methods [15]. In recent years, sign identification of third nonlinear refractive index of materials by Moiré deflectometry technique is found by observing size of Moiré fringes and Moiré fringes spacing curves [16, 17]. This technique avoids the requirement of highly calibrated detectors, but still, the need to use a scanner that is highly sensitive to movements exists. In our recent work, we have proposed a novel Moiré deflectometry technique that not only avoids the use of highly sensitive calibrated detectors but also omits the need for scanner [18].

The presented methods in this paper which are based on scanning (Z-scan) and nonscanning (Moiré deflectometry) techniques have been used to identify the sign of the nonlinear refractive index of Ag nano-particles suspended in water.

\section{Experimental}

2.1. Material Processing. The samples of Ag nano-particles for this study were fabricated by the laser ablation method. The synthesis procedure has been detailed elsewhere [19]. The average size of fabricated nano-particles is $\sim 20 \mathrm{~nm}$ and is obtained through the transmission electron microscopy (TEM) technique, Figure 1. The linear absorption spectra of Ag nanoparticles are shown in Figure 2. Absorption peak happens at near $400 \mathrm{~nm}$. 


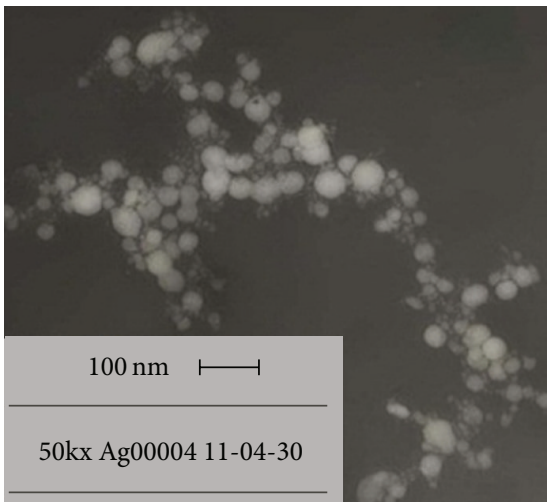

FIGURE 1: Image of silver nanoparticles fabricated during laser ablation technique by method of transmission electron microscopy.

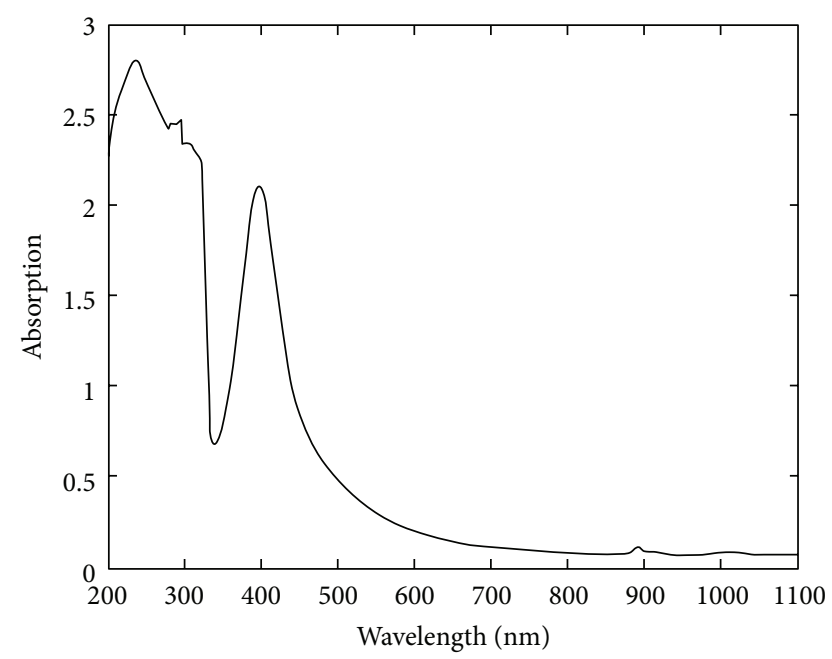

Figure 2: Absorption spectra of Ag nanoparticles.

2.2. Moiré Deflectometry Method. In this technique a Gaussian laser beam with a high intensity as a pump beam is emitted to the sample (Laser2). This beam causes nonlinear effects in the sample which will lead to changes in the refractive index in the sample environment. An expanded beam of low intensity will be emitted perpendicularly on the pump beam (Laser1). The sign of nonlinear refractive index is determined only usign the probe beam without any further calculation. The block diagram of the experiment for Moiré deflectometry method is depicted in Figure 3.

The intensity-dependent refractive index $n$, is defined as [20]:

$$
n=n_{0}+\Delta n=n_{0}+n_{2} I
$$

where $n_{0}$ is the first refractive index, $n_{2}$ is the second order of refractive index, and $I$ is the intensity of the incident pump beam which causes nonlinear effects. The difference between refractive index of interaction zone and other area of sample creates a cylindrical lens which is shown in Figure 4.

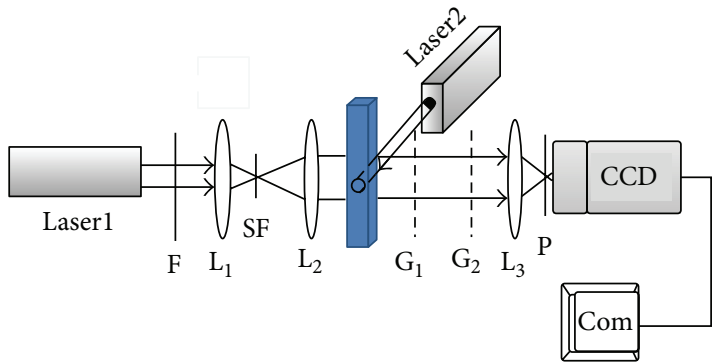

FIGURE 3: The experimental setup of Moiré deflectometry for sign identifying nonlinear refractive index, (a): Laserl (probe laser); $15 \mathrm{~mW}$ He-Ne laser, Laser2 (pump laser); $47 \mathrm{~mW}$ green laser, F; filter, SF; spatial filter, $G_{1}, G_{2}$; grating, $L_{1}, L_{2}$, and $L_{3}$; lenses with the focal lengths of $f_{1}=50 \mathrm{~mm}, f_{2}=150 \mathrm{~mm}$, and $f_{3}=250 \mathrm{~mm}, \mathrm{~S}$ sample, $\mathrm{P}$; the pinhole, CCD; the camera, Com; the computer.

According to lens-makers formula, the focal length of this lens can be written as [21]:

$$
\frac{1}{f}=\left(n-n_{0}\right)\left[\frac{1}{R_{1}}-\frac{1}{R_{2}}\right] \text {, }
$$

where $R_{1}$ and $R_{2}$ are the first and second radius of lens, respectively, and are equal to the pump laser beam radius $R$. By using (1) and (2), the focal length of cylindrical lens created by the nonlinear effects will be

$$
f=\frac{R}{2 n_{2} I} .
$$

Equation (3) shows that concave or convex lenses are generated as a result of negative or positive nonlinear refractive indexes of sample, respectively. Convergence or divergence of probe beam illuminated on the generated cylindrical lens will determine the sign of nonlinear refractive index. The convergence of the beam corresponds to positive $\left(n_{2}>0\right)$ and divergence of the beam corresponds to negative $\left(n_{2}<0\right)$ nonlinear refractive index [18].

2.3. Z-Scan Method. We used the Z-scan method as the second method to investigate sign identification of nonlinear refractive index of silver nano-particles. The experimental setup is shown in Figure 5. In Z-scan method, the scanning starts from a distance far away from the focus (negative $z$ ), and when the sample is brought closer to the focus, the beam irradiance increases leading to self-lens effect in the sample. In negative nonlinearity, a negative self-lens effect occurs prior to focus which tends to collimate the beam and reduces the diffraction leading to a smaller beam at the aperture and an increased transmittance. As the sample crosses the focal plane to the right (positive $z$ ), the diffraction of the beam will, change and the aperture transmittance will be reduced due to the same self-defocusign effect. Therefore, prefocal transmittance maximum (peak) and postfocal transmittance minimum (valley) are the representatives of the negative 


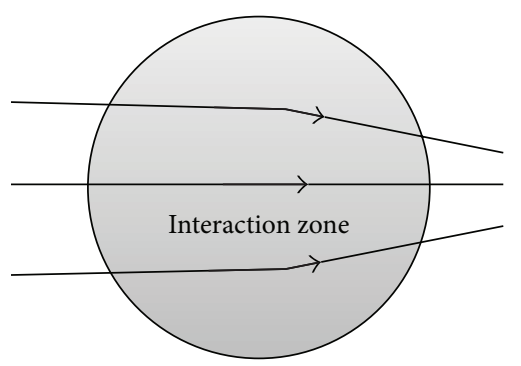

$n_{2}>0$

(a)

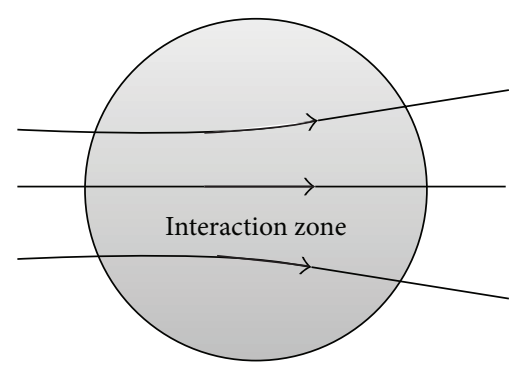

$n_{2}<0$

(b)

FiguRE 4: Interaction zone between guide laser beam and nonlinear environment. (a) A convex lens as a result of positive refractive index. (b) A concave lens as a result of negative refractive index.

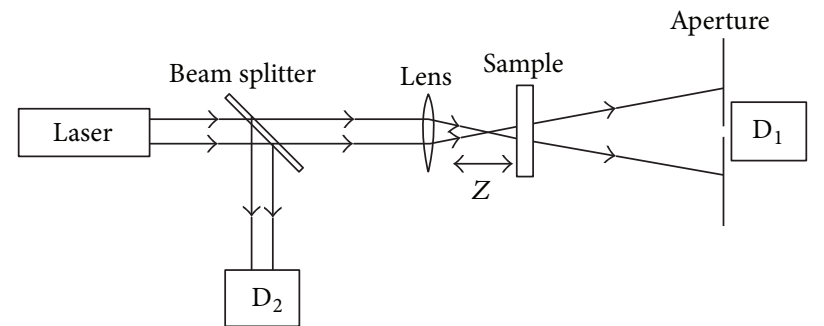

FIGURE 5: The experimental setup for measuring optical nonlinearity by use of the Z-scan technique, and $\mathrm{D}_{1}$ and $\mathrm{D}_{2}$ are detectors for beam intensity detection.

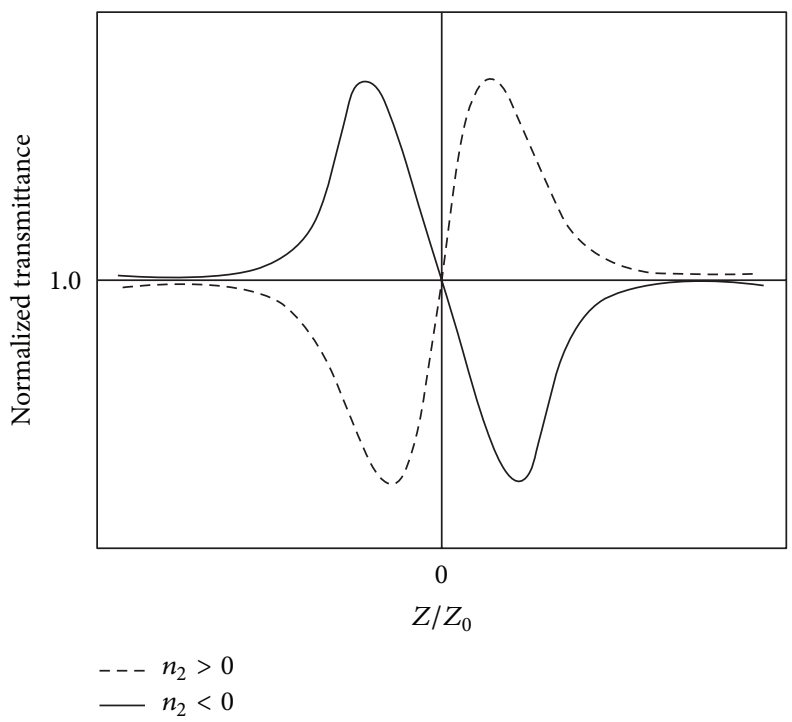

FIGURE 6: Z-scan theoretical curves of the transmittance as a function of $z$.

sign of nonlinear refractive index as shown by the peakvalley configuration. Following the same analogy, the $\mathrm{Z}$ scan signature of a positive nonlinearity will give rise to an opposite valley-peak configuration. Figure 6 depicts these two configurations. Z-scan method requires sensitive and calibrated detector which makes the accuracy of measurements dependent on the accuracy of detector's response.

\section{Experimental Results and Discussions}

We have examined the Moiré deflectometry technique for measuring the nonlinear refractive index in colloidal Ag nano-particles. To identify the sign of nonlinear refractive index of the sample, the experiment was set up as shown in Figure 3; a $15 \mathrm{~mW}$ He-Ne laser beam has been used as a probe 


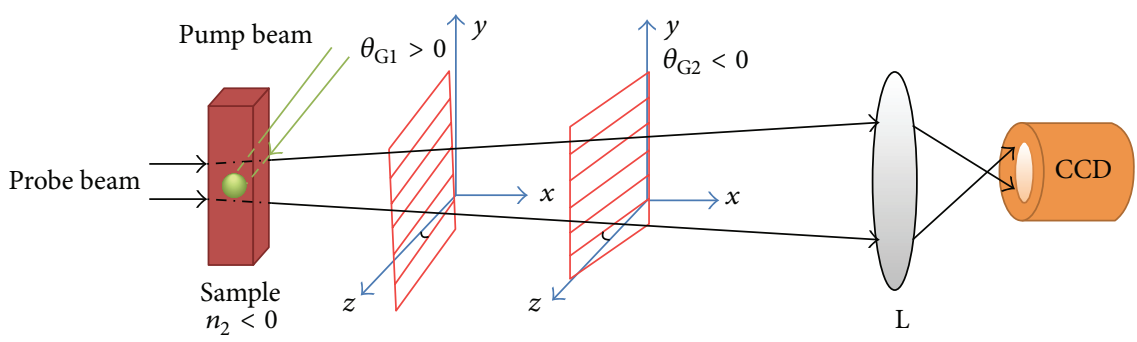

(a)

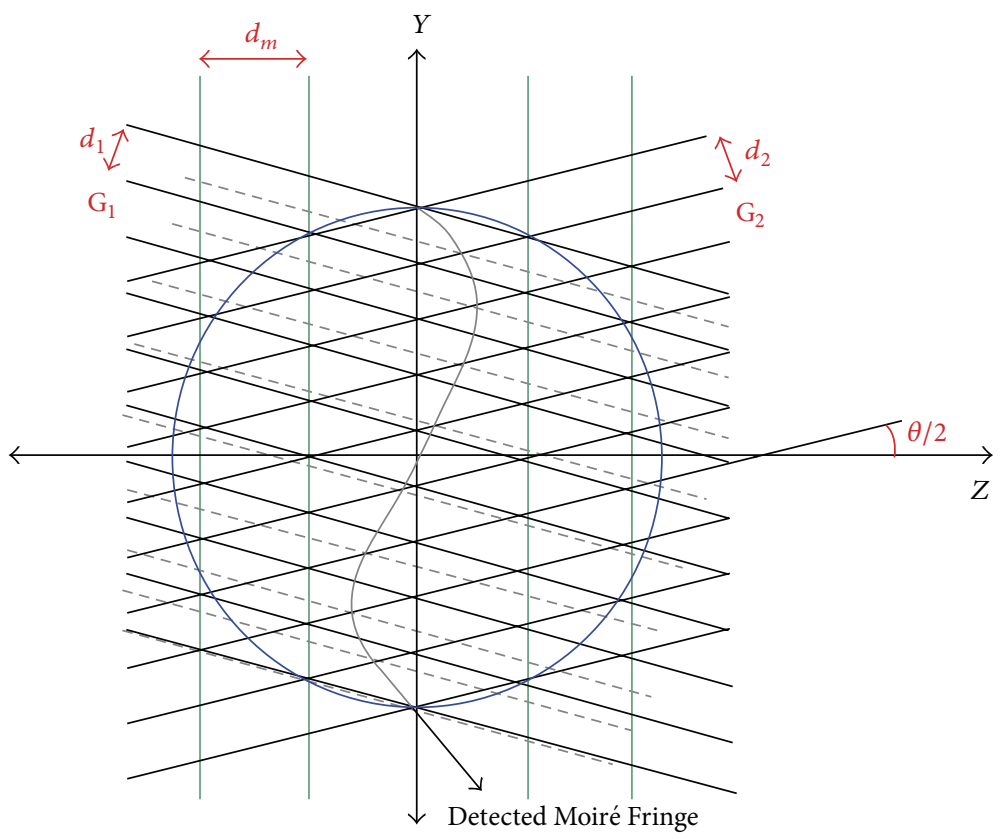

(b)

Figure 7: (a) The experimental setup for $n_{2}<0, \theta_{\mathrm{G} 1}>0$, (b) deflection of Moiré pattern; the red dashed lines show the movement of the grating vector image, in which $\mathrm{G}_{i}$ is grating, $\mathrm{L}$ is lens, and $d_{i}$ and $d_{m}$ are the pitches of grating and Moiré fringes, respectively.

beam, which has been expanded and collimated by lenses $\mathrm{L}_{1}$, $\mathrm{L}_{2}$ and a spatial filter. This beam will then pass the nanoparticles, which is in a Quartz cell with $10 \mathrm{~mm}$ thickness. As the laser beam passes through grating $G_{1}$ and $G_{2}$, Moiré fringe patterns are projected on a CCD camera by lens $\mathrm{L}_{3}$ and recorded by a computer. A $47 \mathrm{~mW}$ second harmonic Nd:YAG laser is used as pump laser, and the beam is emitted to the sample. After generating the thermal gradient in the colloidal nano-particles, the deflection of Moiré fringes will appear [18]. By choosign the appropriate coordinates as shown in Figures 7 to 8 and rotating the grating along the $Z$ axes, we can observe the effect of rotation of the first grating on the Moire fringe patterns. As shown in Figure 9, the deflected Moiré fringes were determined for Ag nano-particles. By changing direction of first grating angle, the deflected Moiré fringes change patterns as shown in Figures 9(b) and 9(c). Finally, by knowing the direction of deflected Moiré fringes and the direction of rotation of first grating angle, the sign of nonlinear refractive index of $\mathrm{Ag}$ nano-particles was found to be negative.
Figure 4 shows the schematic setup for close-aperture Zscan experiment. The excitation source was a second harmonic Nd:YAG laser with a beam power of $55 \mathrm{~mW}$ and was focused onto the sample by a lens with $10 \mathrm{~cm}$ focal length, and the beam waist radius $\left(w_{0}\right)$ was measured to be $14.7 \mu \mathrm{m}$, and the corresponding Rayleigh length was $1.32 \mathrm{~mm}$. The thickness of the quartz cell containing the sample was $1 \mathrm{~mm}$, which was less than the Rayleigh length of the laser beam. Prefocal transmittance maximum (peak), followed by a postfocal transmittance minimum (valley) for Ag nano-particles, is shown in Figure 10.

\section{Conclusion}

As shown in this paper, two different methods can be used to identify the sign of thermal nonlinear refractive index, caused by the interaction of a laser beam with the colloidal silver nano-particles in water solution.

The proposed Moiré deflectometry technique is a nonscanning method, and the sign of refractive index can be 


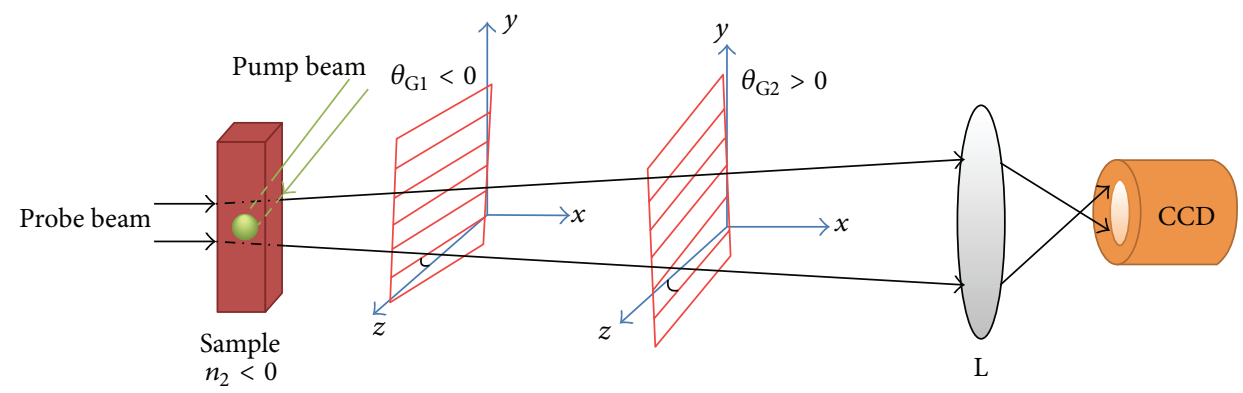

(a)

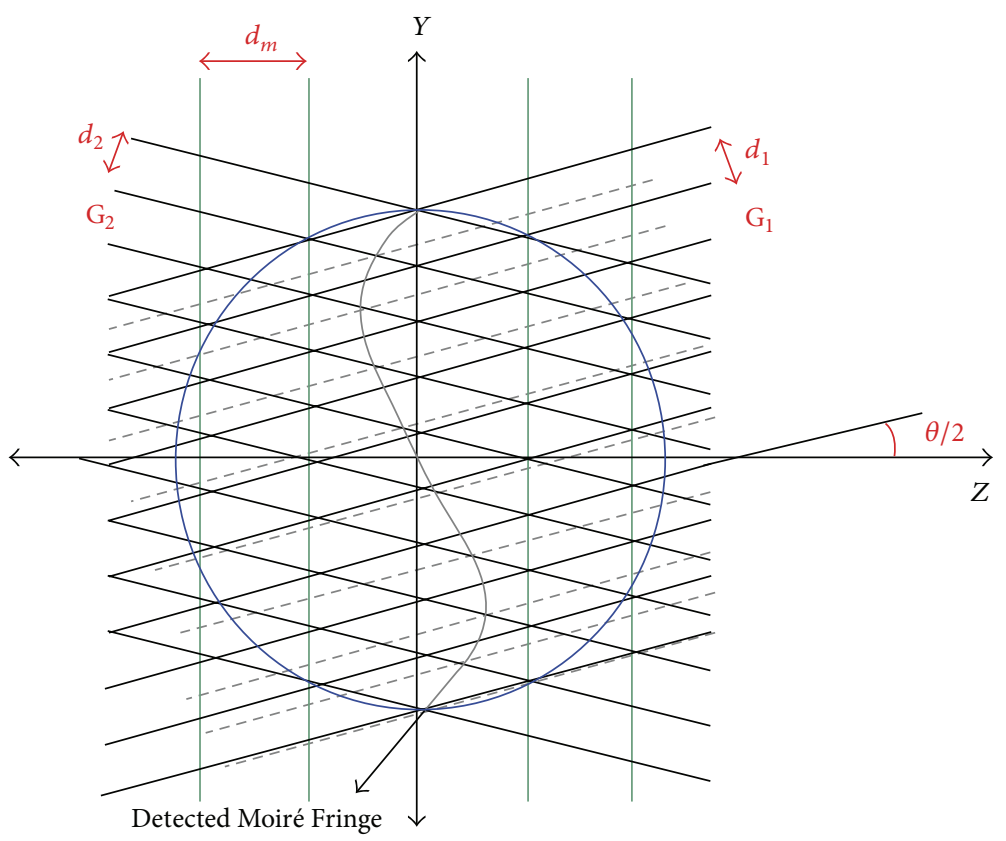

(b)

Figure 8: (a) The experimental setup for $n_{2}<0, \theta_{\mathrm{G} 1}<0$, (b) deflection of Moiré pattern; the red dash lines show the movement of the grating vector image, in which $\mathrm{G}_{i}$ is grating, $\mathrm{L}$ is lens, and $d_{i}$ and $d_{m}$ are the pitches of grating and Moiré fringes, respectively.

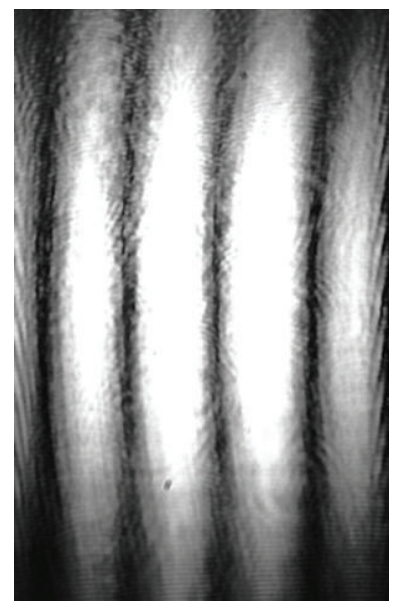

(a)

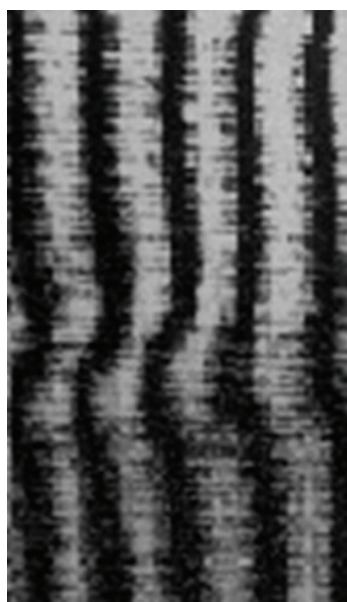

(b)

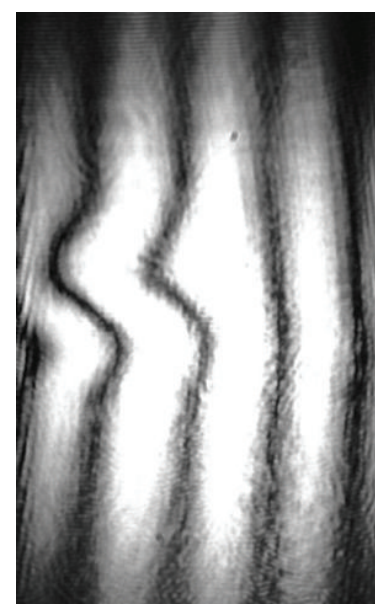

(c)

FIGURE 9: The deflection of Moiré fringes patterns of Ag nano-particles caused by producing refractive index gradient, (a) the Moiré fringes before deflection, (b) the Moiré fringes after deflection, $\theta_{\mathrm{G} 1}>0$, and (c) the Moiré fringes after deflection, $\theta_{\mathrm{G} 1}<0$. 


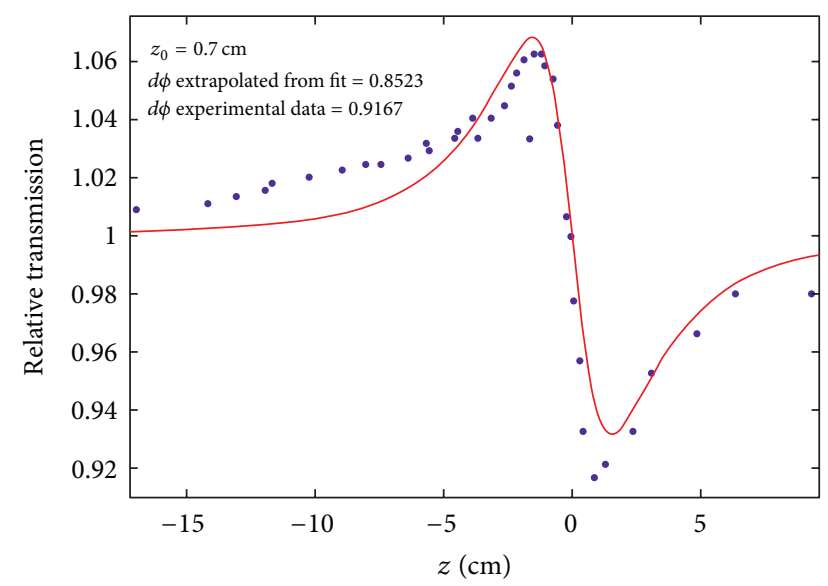

FIGURE 10: Z-scan normalized transmittance with close aperture of colloidal Ag nanoparticles. The solid curve is the theoretical fit to the data.

achieved immediately and in real time. By observing the deflection of Moiré fringes, the sign of nonlinear refractive index can be determined. This method is simple, fast, and not sensitive to environment noise and vibration. Also, it does not need calibration or analysis of fringe patterns. Furthermore, the closed aperture of Z-scan technique was used to identify the sign of refractive index of colloidal Ag nano-particles. The Moire deflectometry and Z-scan methods showed that the sign of nonlinear refractive index of colloidal Ag nanoparticles is negative.

\section{References}

[1] R. A. Ganeev, A. I. Ryasnyansky, S. R. Kamalov, M. K. Kodirov, and T. Usmanov, "Nonlinear susceptibilities, absorption coefficients and refractive indices of colloidal metals," Journal of Physics D, vol. 34, no. 11, pp. 1602-1611, 2001.

[2] J. Zyss, Molecular Nonlinear Optics: Materials, Physics, and Devices, Academic Press, Boston, Mass, USA, 1994.

[3] D. Lupo, "Principles and applications of nonlinear optical materials. Edited by R. W. Munn and C. N. Ironside, Chapmann \& Hall, London 1992, 257 pp., hardcover,\# 69, ISBN 0-7514-00858," Advanced Materials, vol. 5, no. 10, pp. 772-773, 1993.

[4] M. Sheik-Bahae, A. A. Said, T. Wei, D. J. Hagan, and E. W. Van Stryland, "Sensitive measurement of optical nonlinearities using a single beam," IEEE Journal of Quantum Electronics, vol. 26, no. 4, pp. 760-769, 1990.

[5] M. Sheik-bahae, A. A. Said, and E. W. Van Stryland, "High-sensitivity, single-beam $n_{2}$ measurements," Optics Letters, vol. 14, no. 17, pp. 955-957, 1989.

[6] M. Sheik-Bahae, J. Wang, R. DeSalvo, D. J. Hagan, and E. W. Van Stryland, "Measurement of nondegenerate nonlinearities using a two-color Z scan," Optics Letters, vol. 17, no. 4, pp. 258-260, 1992.

[7] M. Sheik-Bahae, A. Said, D. J. Hagan, M. J. Soileau, and E. W. Van Stryland, "Nonlinear refraction and optical limiting in "thick" media," Optical Engineering, vol. 30, no. 8, pp. 1228-1235, 1991.
[8] S. S. Lin, "Optical properties of $\mathrm{TiO}_{2}$ nanoceramic films as a function of N-Al co-doping," Ceramics International, vol. 35, no. 7, pp. 2693-2698, 2009.

[9] S. S. Lin, Y. H. Hung, and S. C. Chen, "Optical properties of $\mathrm{TiO}_{2}$ thin films deposited on polycarbonate by ion beam assisted evaporation," Thin Solid Films, vol. 517, no. 16, pp. 4621-4625, 2009.

[10] S. S. Lin, Y. H. Hung, and S. C. Chen, "The properties of $\mathrm{TiO}_{2}$ nanoceramic films prepared by electron beam evaporation," Journal of Nanoscience and Nanotechnology, vol. 9, no. 6, pp. 3599-3605, 2009.

[11] S. S. Lin, S. C. Chen, and Y. H. Hung, " $\mathrm{TiO}_{2}$ nanoceramic films prepared by ion beam assisted evaporation for optical application," Ceramics International, vol. 35, no. 4, pp. 1581-1586, 2009.

[12] S. S. Lin and D. K. Wu, "Enhanced optical properties of $\mathrm{TiO}_{2}$ nanoceramic films by oxygen atmosphere," Journal of Nanoscience and Nanotechnology, vol. 10, no. 2, pp. 1099-1104, 2010.

[13] S. Rasouli and K. Jamshidi-Ghaleh, "Erratum to "Nonlinear refraction measurements of materials using the Moiré deflectometry" [K. Jamshidi-Ghaleh, N. Mansour, Opt. Commun. 234 (2004) 419] (Optics Communications (2004) 234 (419)," Optics Communications, vol. 284, no. 5, pp. 1481-1482, 2011.

[14] Z. Karny and O. Kafri, "Refractive-index measurements by moire deflectometry," Applied Optics, vol. V 21, no. 18, pp. 33263328, 1982.

[15] O. Kafri and I. Glatt, The Physics of Moiré Metrology, Wiley, New York, NY, USA, 1989.

[16] K. J. Ghaleh and N. Mansour, "Nonlinear refraction measurements of materials using the Moiré deflectometry," Optics Communications, vol. 234, no. 1-6, pp. 419-425, 2004.

[17] S. Rasouli, H. Ghasemi, M. T. Tavassoly, and H. R. Khalesifard, "Application of "parallel" Moiré deflectometry and the single beam Z-scan technique in the measurement of the nonlinear refractive index," Applied Optics, vol. 50, no. 16, pp. 2356-2360, 2011.

[18] A. Granmayeh Rad, K. Madanipour, A. Koohian, and N. Taheri, "Sign identification of nonlinear refractive index of colloidal nanoparticles by Moiré deflectometry technique," Journal of the European Optical Society, vol. 7, Article ID 12034, 5 pages, 2012.

[19] A. Granmayeh Rad, H. Abbasi, and K. Golyari, "Fabrication and nonlinear refractive index measurement of colloidal silver nanoparticles," International Journal of Applied Physics and Mathematics, vol. 2, no. 2, pp. 135-139, 2012.

[20] R. W. Boyd, Nonlinear Optics, chapter 4, Academic Press, New York, NY, USA, 2nd edition, 2003.

[21] E. Hecht, Optics, chapter 5, Addison Wesley, Reading, Mass, USA, 4th edition, 2002. 

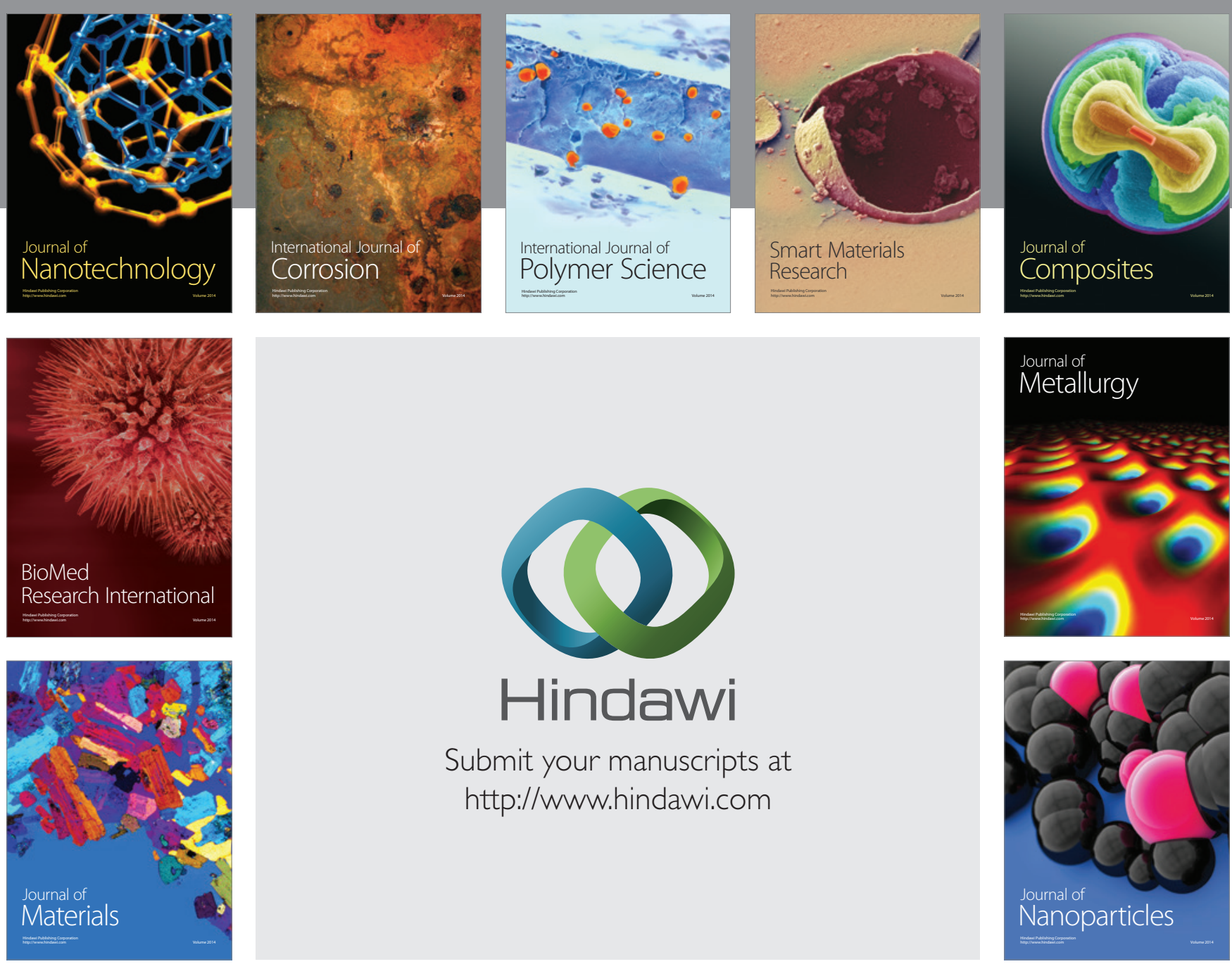

Submit your manuscripts at http://www.hindawi.com
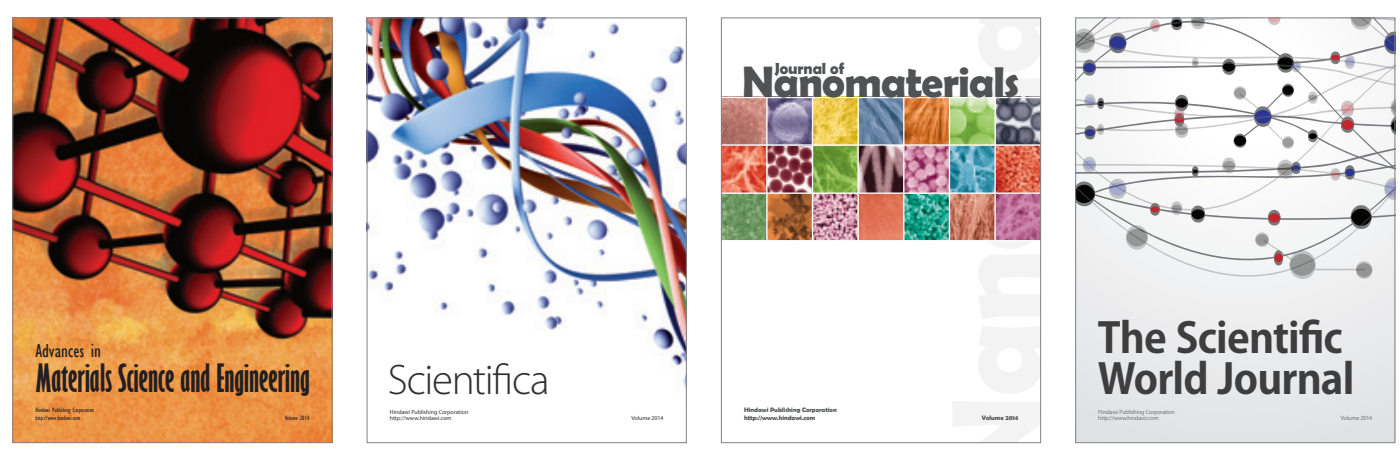

\section{The Scientific World Journal}
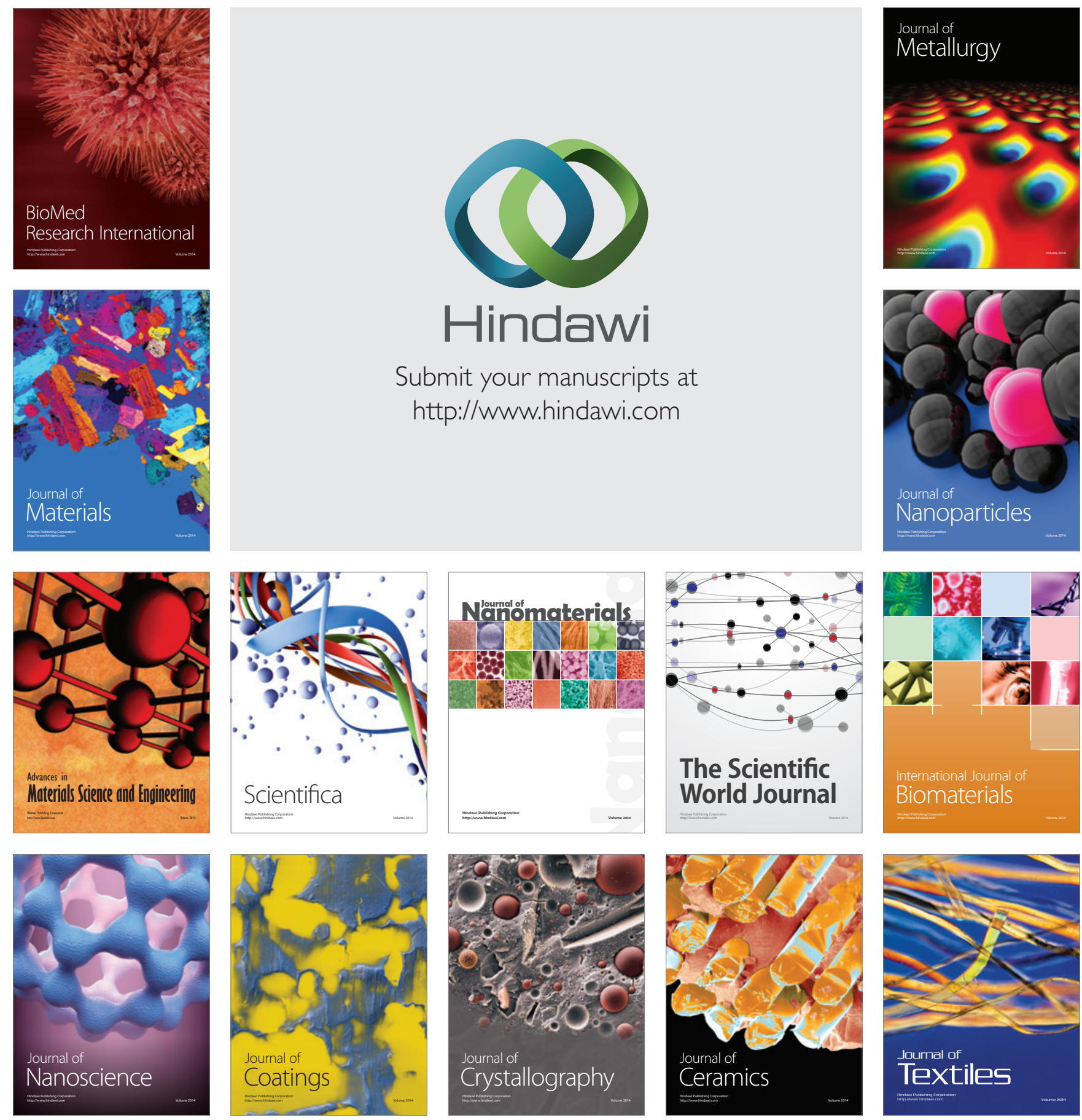